\title{
D-galactose-induced mitochondrial DNA oxidative damage in the auditory cortex of rats
}

\author{
ZHENGDE DU ${ }^{1,2^{*}}$, QIONG YANG ${ }^{1,2^{*}}$, TAO ZHOU ${ }^{3 *}$, LIN LIU $^{4}$, SHUO LI $^{1,2}$, \\ SHIXIONG $\mathrm{CHEN}^{5}$ and CHUNSHENG GAO ${ }^{1,2}$
}

\author{
${ }^{1}$ Department of Otorhinolaryngology, Nanshan Affiliated Hospital of Guangdong Medical College; \\ ${ }^{2}$ The Chinese University of Hong Kong-Shenzhen Nanshan Hospital Joint Otorhinolaryngology, \\ Head and Neck Surgery Training Centre cum Microsurgical and Endoscopic Skills Laboratory, Shenzhen, \\ Guangdong 518052; ${ }^{3}$ Department of Otorhinolaryngology, Union Hospital, Tongji Medical College, \\ Huazhong University of Science and Technology, Wuhan, Hubei 430022; ${ }^{4}$ College of Pharmacy, Guangxi Medical University, \\ Nanning, Guangxi Zhuang Autonomous Region 530021; ${ }^{5}$ Shenzhen Institutes of Advanced Technology, \\ Chinese Academy of Sciences, Shenzhen, Guangdong 518055, P.R. China
}

Received October 21, 2013; Accepted June 2, 2014

DOI: $10.3892 / \mathrm{mmr} .2014 .2653$

\begin{abstract}
Chronic administration of D-galactose (D-gal) is a useful method for establishing a model of natural aging in the auditory system. Previous studies have demonstrated that NADPH oxidases (NOXs) may be an important source of reactive oxygen species (ROS) in the peripheral auditory system (PAS) and cause an increase in mitochondrial DNA (mtDNA) common deletion (CD) levels in the PAS and central auditory system (CAS) of rats with D-gal-induced aging. However, the source of the ROS in the CAS and the mechanisms of age-related hearing loss (ARHL) have yet to be elucidated. In the present study, male Sprague Dawley rats were administered a daily injection of D-gal (150, 300 and $500 \mathrm{mg} / \mathrm{kg}$, respectively) for eight weeks. All three doses of D-gal caused a significant increase in the expression of NOX2, 8-hydroxy-2-deoxyguanosine, a biomarker of DNA oxidative damage, and uncoupling protein 2 , together with
\end{abstract}

Correspondence to: $\mathrm{Dr}$ Chunsheng Gao, Department of Otorhinolaryngology, Nanshan Affiliated Hospital of Guangdong Medical College, 89 Taoyuan Road, Nanshan, Shenzhen, Guangdong 518052, P.R. China

E-mail: entcsgao@163.com

*Contributed equally

Abbreviations: ARHL, age-related hearing loss; CAS, central auditory system; CD, common deletion; D-gal, D-galactose; mtDNA, mitochondrial DNA; NOX, NADPH oxidase; PAS, peripheral auditory system; ROS, reactive oxygen species; T-AOC, total antioxidant capability; UCP, uncoupling protein; 8-OHdG, 8-hydroxy-2-deoxyguanosine

Key words: D-galactose, auditory cortex, oxidative damage, mitochondria, age-related hearing loss a decrease in the mitochondrial total antioxidant capabilities in the auditory cortex, as compared with the control rats (injected daily with the same volume of $0.9 \%$ saline for eight weeks). The levels of the mtDNA CD were also increased in the auditory cortex of the D-gal-induced aging rats. These findings suggest that both NOX-and mitochondria-associated ROS generation may contribute to mtDNA oxidative damage in the auditory cortex of the CAS of D-gal-induced aging rats. This study may provide novel insight into the development of ARHL.

\section{Introduction}

Aging is the result of complex changes in the structure and function of molecules, cells, tissues and whole body systems. Age-related hearing loss (ARHL), also known as presbycusis, is believed to result from age-related degeneration of the peripheral and central components of the auditory system $(1,2)$. Investigations into ARHL in humans is limited due to the inaccesibility of auditory system tissue and the complexity of the genetic and environmental background of individuals with hearing loss. Numerous animal models have been established in order to facilitate research into the molecular mechanisms of ARHL. Among them, an animal model using the chronic administration of D-galactose (D-gal) is widely used for studying the mechanisms of ARHL (3-11). These animals exhibit increased oxidative stress and mitochondrial DNA (mtDNA) common deletion (CD) in the peripheral and central auditory system (PAS and CAS, respectively); however, the source of the causative reactive oxygen species (ROS) in the CAS has not been fully investigated.

NADPH oxidases (NOXs) are one of the main ROS-generating sites. It is now clear that NOXs are not restricted to the immune system and that alternative isoforms may be active in numerous cell types as essential components of cellular signalling, gene expression regulation and cell differentiation. These enzymes are able to transport electrons 
across the plasma membrane, generating superoxide and other downstream ROS (12). The expression of NOX3 is higher in the PAS than that in any other tissue (13). In a previous study (5), it was demonstrated that NOX3 may be an important source of ROS in the PAS of rats with D-gal-induced aging and that chronic injection of D-gal could increase NOX3-dependent oxidative stress, mitochondrial damage and apoptosis in the PAS. NOX2 is not restricted to phagocytic cells, but is present in numerous non-phagocytic cells and tissues, including neurons of the CAS $(14,15)$. The effects of NOX2 in the auditory cortex of the CAS of rats with D-gal-induced aging have yet to be elucidated.

Mitochondria are another site of predominant ROS generation in cells (16). Mitochondrial ROS generation is sensitive to the proton-motive force across the mitochondrial inner membrane produced by the electron transport chain, and mild uncoupling caused by the activation of uncoupling protein 2 (UCP2) may cause a reduction in the proton-motive force, attenuate mitochondrial ROS generation and protect cells against ROS-related cellular damage (17). It is therefore hypothesised that an overexpression of UCP2 may indirectly increase mitochondrial ROS generation. The expression of $\mathrm{UCP} 2$ in the CAS of rats with D-gal-induced aging, however, is unclear.

In the present study, the expression of NOX2, UCP2 and 8-hydroxy-2-deoxyguanosine (8-OHdG), a biomarker of DNA oxidative damage $(18,19)$, as well as the mitochondrial total antioxidant capabilities (T-AOCs) and the levels of the mtDNA CD, were investigated in the auditory cortex of the CAS in a rat model with D-gal-induced aging. It was hypothesised that NOX- and mitochondria-dependent ROS generation and mtDNA oxidative damage may be primary etiological events in the degeneration of the CAS of rats with D-gal-induced aging.

\section{Material and methods}

Animals and treatments. Eighty-eight one-month-old male Sprague Dawley rats were obtained from the Experimental Animal Centre of Guangxi Medical University (Nanning, China). The rats were individually housed in temperature-controlled $\left(20-22^{\circ} \mathrm{C}\right)$ conditions with a 12 -h light/dark cycle, with free access to food and water. The body weight of each of the rats was monitored throughout the experiment as an indicator of health. Injections of D-gal to induce aging were administered according to established methodology (9). Following acclimation for two weeks, the rats were randomly divided into four groups ( $n=22$ for each group), in which they were administered daily doses of D-gal (Sigma, St. Louis, MO, USA) or saline by subcutaneous injection for eight weeks. The three D-gal groups were referred to as the low- $(150 \mathrm{mg} / \mathrm{kg})$, medium- $(300 \mathrm{mg} / \mathrm{kg})$ and high$(500 \mathrm{mg} / \mathrm{kg}$ ) dose groups and the fourth group was a control group (administered $0.9 \%$ saline at the same volume). At the end of the eight-week protocol, the rats were sacrificed by a terminal intraperitoneal injection of ketamine $(30 \mathrm{mg} / \mathrm{kg})$ and an intramuscular injection of chloropromazine $(15 \mathrm{mg} / \mathrm{kg})$. The auditory cortex was dissected and used for total RNA, genomic DNA and protein extraction and the examination of mitochondrial T-AOCs. Alternatively, the rats were perfused with $4 \%$ paraformaldehyde for immunohistochemical analysis. All experiments were carried out in strict accordance with the recommendations in the Guide for the Care and Use of Laboratory Animals of the National Institutes of Health. The protocol was approved by the Committee on the Ethics of Animal Experiments of Guangxi Medical University.

RNA preparation and $S Y B R^{\circledR}$ Green quantitative polymerase chain reaction $(q P C R)$. The mRNA expression levels of NOX2 and UCP2 were determined using a SYBR Green qPCR assay (Invitrogen Life Technologies, Carlsbad, CA, USA). Following the final injection of D-Gal, 24 rats $(n=6$ per group) were sacrificed, and both sides of the auditory cortex from each rat were rapidly removed. One side of the auditory cortex was used for RNA extraction, and the other side was used for mtDNA analysis. Total RNA was extracted with TRIzol ${ }^{\circledR}$ reagent (Takara, Dalian, China) according to the manufacturer's instructions. cDNA was reverse transcribed using a PrimeScript RT Reagent kit (Takara). The RNA and cDNA of each sample were analysed using a GeneQuant Pro DNA/RNA Calculator (Biochrom, Cambridge, UK) to assess the concentrations and purity. The cDNA samples were stored at $-20^{\circ} \mathrm{C}$ until required. qPCR was performed by applying the SYBR Green qPCR technology with the use of a StepOnePlus ${ }^{\mathrm{TM}}$ Real-Time PCR system (Applied Biosystems, Foster City, CA, USA). The primer pairs for NOX2, UCP2 and $\beta$-actin (as an internal standard) were as follows: NOX2 forward, 5'-ACATTTTCGTCAAGCGTCCC-3' and NOX2 reverse, 5'-CCCAGCTCCCACTAACATCA-3'; UCP2 forward, 5'-TGCTGGGCACCATCCTAACC-3' and UCP2 reverse, 5'-CCTGGAAGCGGACCTTTACC-3'; $\beta$-actin forward, 5'-CCTGGAGAAGAGCTATGAGC-3' and $\beta$-actin reverse, 5'-ACAGGATTCCATACCCAGG-3'. The amplification conditions were as follows: $30 \mathrm{sec}$ at $95^{\circ} \mathrm{C}$, then 40 cycles of $5 \mathrm{sec}$ at $95^{\circ} \mathrm{C}, 30 \mathrm{sec}$ at $60^{\circ} \mathrm{C}$ and $30 \mathrm{sec}$ at $72^{\circ} \mathrm{C}$. The amplification of $\beta$-actin as an internal standard was used to normalise the relative gene expression levels. A melting curve analysis was performed for each gene, and the specificity and integrity of the PCR products were confirmed by the presence of a single peak. The relative expression levels were calculated from the differences in the cycle threshold $(\mathrm{Ct})$ values between the target mRNA and $\beta$-actin. The change in the relative mRNA levels between the experimental group and the control group was analysed using the $2^{-\Delta \Delta} \mathrm{Ct}$ method, as previously reported (20).

Immunohistochemical analysis. The protein levels of NOX2 and the expression of $8-\mathrm{OHdG}$ were determined by immunohistochemistry. Sixteen rats ( $n=4$ per group) were sacrificed, and the brains were removed and fixed with $4 \%$ buffered paraformaldehyde overnight, dehydrated and embedded in paraffin wax. Serial sections of the brainstem were subsequently cut at a thickness of $5 \mu \mathrm{m}$ at the level of the auditory cortex. The sections were then deparaffinised in xylene and rehydrated through graded concentrations of ethanol. The samples were incubated with anti-NOX2 (diluted 1:200; Boster Biological Technology Co., Ltd., Wuhan, China) and anti-8-OHdG (diluted 1:4,000; Abcam, Cambridge, MA, USA) antibodies overnight at $4{ }^{\circ} \mathrm{C}$. Following washes 
in phosphate-buffered saline, the slides were incubated with fluorescein isothiocyanate-conjugated anti-rabbit and Cy3-conjugated anti-mouse secondary antibodies (diluted 1:200; Boster Biological Technology Co., Ltd.) for $30 \mathrm{~min}$ at room temperature and the nuclei were counterstained using DAPI staining solution (Beyotime Institute of Biotechnology, Haimen, China) for $5 \mathrm{~min}$ at room temperature. Images were captured using a laser scanning confocal microscope (Nikon, Tokyo, Japan) and analysed using Image-Pro Plus 6.0 software (Media Cybernetics, Inc., Rockville, MD, USA). As a negative control, sections were treated in the same manner but without the incubation with primary antibody.

Western blot analysis. The protein levels of UCP2 in the auditory cortex were determined using western blot analysis. Twenty-four rats ( $n=6$ per group) were sacrificed, and both sides of the auditory cortex from each rat were dissected. The total protein was extracted using radioimmunoprecipitation assay buffer (Beyotime Institute of Biotechnology) according to the manufacturer's instructions. Protein concentrations were determined using an Enhanced Bicinchoninic Acid Protein Assay kit (Beyotime Institute of Biotechnology). Protein lysate $(30 \mu \mathrm{g})$ was separated by $12 \%$ SDS-PAGE and transferred to polyvinylidene difluoride membranes. The membranes were incubated for $1 \mathrm{~h}$ in a blocking solution [Tris-buffered saline (TBS) containing 5\% skimmed milk], then washed briefly in TBS and incubated overnight at $4{ }^{\circ} \mathrm{C}$ with the appropriate dilution of primary antibodies: Anti-UCP2 (diluted 1:500; Abcam) or anti- $\beta$-actin (diluted 1:1,000; Bioworld Technology, Inc., Minneapolis, MN, USA). The membranes were then washed to remove excess primary antibody and incubated for $1 \mathrm{~h}$ at room temperature with the appropriate horseradish peroxidase-conjugated secondary antibody (diluted 1:5,000; Santa Cruz Biotechnology, Inc., Santa Cruz, CA, USA). The membranes were visualised by the enhanced chemiluminescence method using BeyoECL Plus (Beyotime Institute of Biotechnology) reagent. A quantification of the detected bands was performed using Image-Pro Plus 6.0 software. $\beta$-actin was used as an internal control.

Mitochondrial T-AOC determination. Twenty-four rats $(\mathrm{n}=6$ per group) were sacrificed, and both sides of the auditory cortex from each rat were dissected. Mitochondria in the auditory cortex were quickly extracted using a Tissue Mitochondria Isolation kit (Beyotime Institute of Biotechnology) and subsequently used for the analysis of the T-AOCs. Mitochondrial T-AOCs in the auditory cortex were detected using a Total Antioxidant Capacity Assay kit in combination with the fluorescence recovery after photobleaching method, according to the manufacturer's instructions (Beyotime Institute of Biotechnology).

DNA isolation and TaqMan ${ }^{\circledR} q P C R$. Total DNA was extracted using the Genomic DNA Purification kit (Tiangen Biotech Co., Ltd, Beijing, China) according to the manufacturer's instructions. The DNA concentration of each specimen was measured using the GeneQuant Pro DNA/RNA Calculator and the mtDNA CD levels were determined using a TaqMan qPCR assay. The displacement (D)-loop in the non-coding

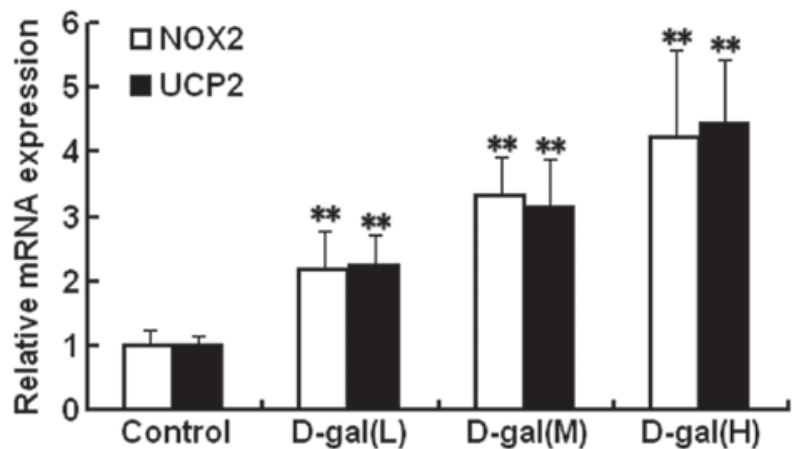

Figure 1. Quantitative analysis of NOX2 and UCP2 mRNA expression in the auditory cortex of rats in the different groups. The mRNA levels of NOX2 and UCP2 were significantly increased in the D-gal-treated rats as compared with those in the control rats. Data are expressed as the mean \pm standard deviation of six rats per group. ${ }^{* *} \mathrm{P}<0.01$ versus the control group. D-gal (L), low-dose D-gal group; D-gal (M), medium-dose D-gal group; D-gal (H), high-dose D-gal group; NOX2, NADPH oxidase; UCP2, mitochondrial uncoupling protein 2; D-gal, D-galactose.

region of mtDNA, representing the conserved segment of mtDNA, was used as a measure of copy number. Primers and probes for the mtDNA D-loop and the mtDNA CD were designed as previously described (21). The PCR amplification was performed on a StepOnePlus Real-Time PCR system in a 20- $\mu 1$ reaction volume consisting of $10 \mu \mathrm{l} 2 \mathrm{X}$ TaqMan PCR mix (Takara), $0.4 \mu 1$ 50X ROX ${ }^{\mathrm{TM}}$ reference dye, $0.4 \mu \mathrm{l}$ each forward and reverse primer $(10 \mu \mathrm{M}), 0.2 \mu \mathrm{l}$ each probe $(10 \mu \mathrm{M}), 4 \mu \mathrm{l}$ sample DNA (10 ng/ $\mu \mathrm{l})$ and $4.6 \mu \mathrm{l}$ distilled water. The cycling conditions consisted of an initial phase at $95^{\circ} \mathrm{C}$ for $30 \mathrm{sec}$, then 40 cycles at $95^{\circ} \mathrm{C}$ for $5 \mathrm{sec}$ and at $60^{\circ} \mathrm{C}$ for $30 \mathrm{sec}$. The cycle number at which a significant increase in the normalised fluorescence was first detected was designated as the $\mathrm{Ct}$ value. The ratio of the mtDNA CD to the mtDNA was calculated by the formula $\Delta \mathrm{Ct}=\left(\mathrm{Ct}_{\mathrm{mtDNA} \text { deletion }}-\mathrm{Ct}_{\mathrm{mtDNA} \mathrm{D} \text {-loop }}\right)$. The relative expression (RE) was used to indicate the factorial difference in the deletions between the experimental and control groups. The RE was calculated according to the $2^{-\Delta \Delta} \mathrm{Ct}$ method, where $\Delta \Delta \mathrm{Ct}=\Delta \mathrm{Ct}_{\mathrm{mtDNA} \text { deletion in experimental group }}-\Delta \mathrm{Ct}_{\mathrm{mtDNA} \text { deletion in control group }}$.

Statistical analysis. The data are presented as the mean \pm standard deviation. The analysis was performed using SPSS 13.0 software (SPSS, Inc., Chicago, IL, USA). Statistical significance was tested by a one-way analysis of variance. The least significant difference post hoc test was used to evaluate the differences between groups. $\mathrm{P}<0.05$ was considered to indicate a statistically significant difference.

\section{Results}

D-gal induces an increase in NOX2 and UCP2 mRNA levels. To investigate the mRNA levels of NOX2 and UCP2 in the auditory cortex, SYBR Green qPCR was performed. As shown in Fig. 1, the mRNA levels of NOX2 and UCP2 were significantly higher in the D-gal-treated groups as compared with those in the control group $(\mathrm{P}<0.01)$.

D-gal induces an increase in NOX2 and 8-OHdG protein expression. Immunohistochemical analysis was performed 
A

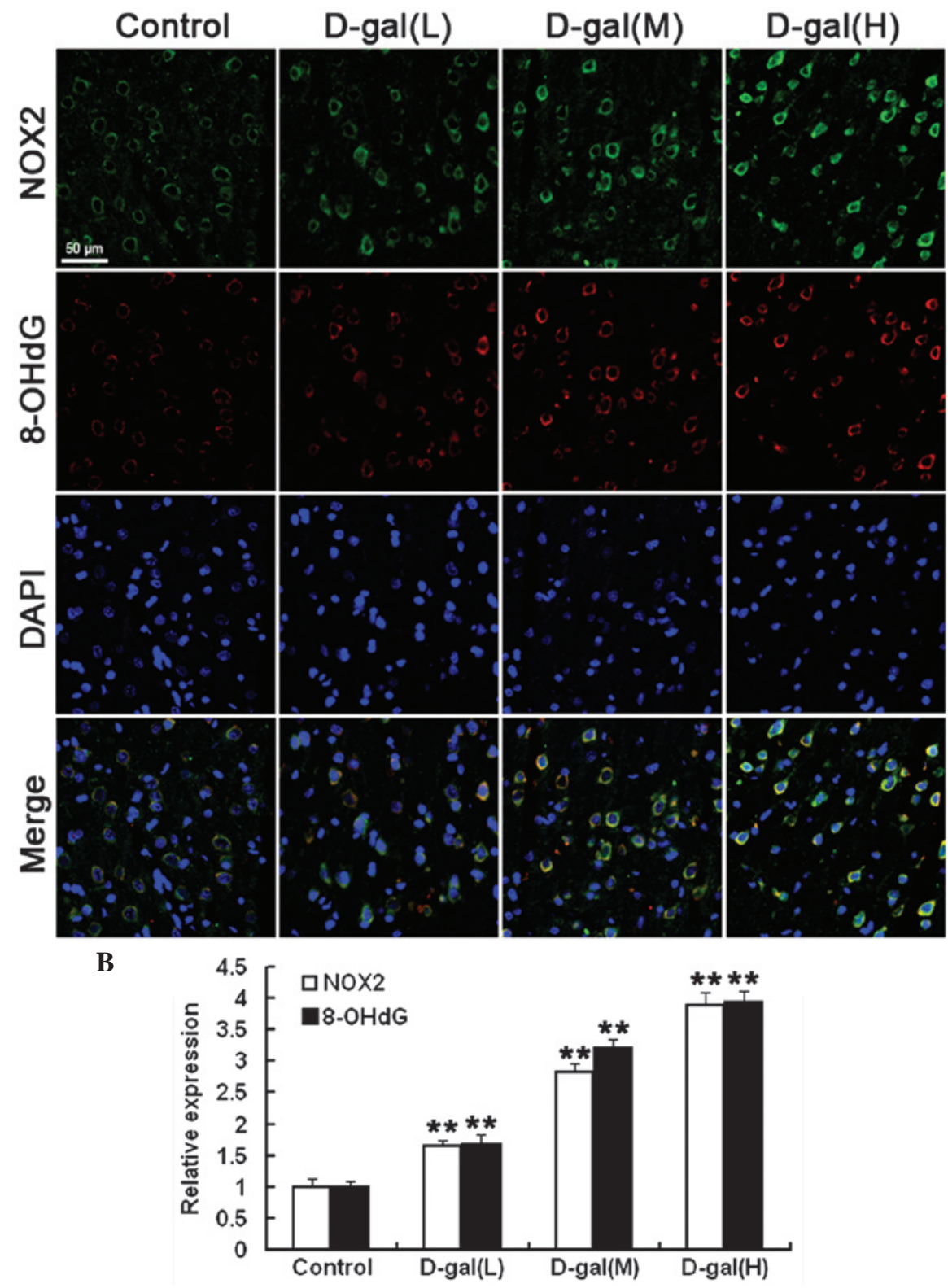

Figure 2. Protein levels of NOX2 and 8-OHdG expression in the auditory cortex of rats in the different groups. (A) Triple staining of rat brain sections with anti-NOX2 (green) and anti-8-OHdG (red) antibodies and DAPI for the staining of cellular nuclei (blue). The expression of 8-OHdG was predominantly localised to the cytoplasm of the cells. Scale bar, $50 \mu \mathrm{m}$; magnification, x600. (B) Quantitative assessment of NOX2 and 8-OHdG expression in the auditory cortex. NOX2 and 8-OHdG expression in the auditory cortex was significantly increased in the D-gal-treated rats as compared with that in the control rats. Data are expressed as the mean \pm standard deviation of four rats per group. ${ }^{* *} \mathrm{P}<0.01$ versus the control group. D-gal (L), low-dose D-gal group; D-gal (M), medium-dose D-gal group; D-gal (H), high-dose D-gal group; NOX2, NADPH oxidase; 8-OHdG, 8-oxo-2'-deoxyguanosine; D-gal, D-galactose.

in order to investigate the protein levels of NOX2 and 8-OHdG expression in the auditory cortex. As shown in Fig. 2A and B, the NOX2 and 8-OHdG expression in the auditory cortex was significantly increased in the D-gal-treated groups as compared with that in the control group. Furthermore, the expression of 8-OHdG was predominantly localised to the cytoplasm of the cells in the auditory cortex (Fig. $2 \mathrm{~A})(\mathrm{P}<0.01)$.

D-gal induces an increase in UCP2 protein expression. Western blot analysis was performed in order to investigate the protein levels of UCP2 in the auditory cortex. As shown in Fig. $3 \mathrm{~A}$ and $\mathrm{B}$, the protein levels of UCP2 were significantly higher in the D-gal-treated groups as compared with those in the control group $(\mathrm{P}<0.01)$.
D-gal induces a decrease in mitochondrial T-AOC. Mitochondrial T-AOC was analysed in the auditory cortex. As shown in Fig. 4, the mitochondrial T-AOCs were significantly decreased in the D-gal-treated groups as compared with those in the control group $(\mathrm{P}<0.01)$.

D-gal induces increased levels of the mtDNA CD. TaqMan qPCR assay was used to investigate the levels of the mtDNA CD in the auditory cortex. The dual-labelled fluorescent DNA probe was designed to specifically recognise the fusion sequence, which was present only in mutant mtDNA that harboured the CD. As shown in Fig. 5, the accumulation of the mtDNA CD was significantly higher in the D-gal-treated groups as compared with that in the control group $(\mathrm{P}<0.01)$. 
A

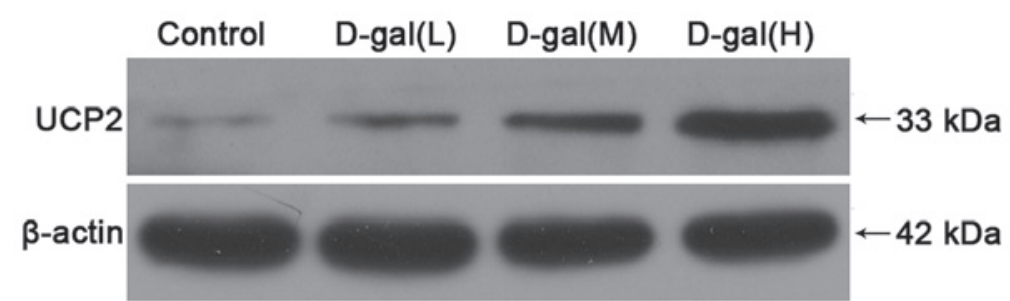

B

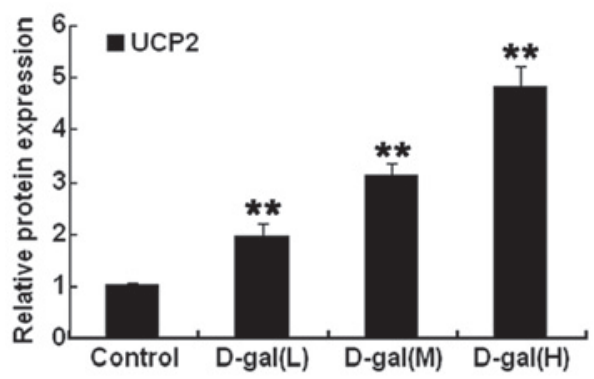

Figure 3. Western blot and densitometric analysis of UCP2 in the auditory cortex of rats in the different groups. (A) Representative western blots showing the expression levels of UCP2 in the different groups. (B) The relative abundance of UCP2 protein was significantly increased in the D-gal-treated groups compared with that in the control group. Data are expressed as the mean \pm standard deviation of six rats per group. ${ }^{* *} \mathrm{P}<0.01$ versus the control group. $\mathrm{D}$-gal (L), low-dose D-gal group; D-gal (M), medium-dose D-gal group; D-gal (H), high-dose D-gal group; UCP2, mitochondrial uncoupling protein 2; kDa, kilodaltons; D-gal, D-galactose.

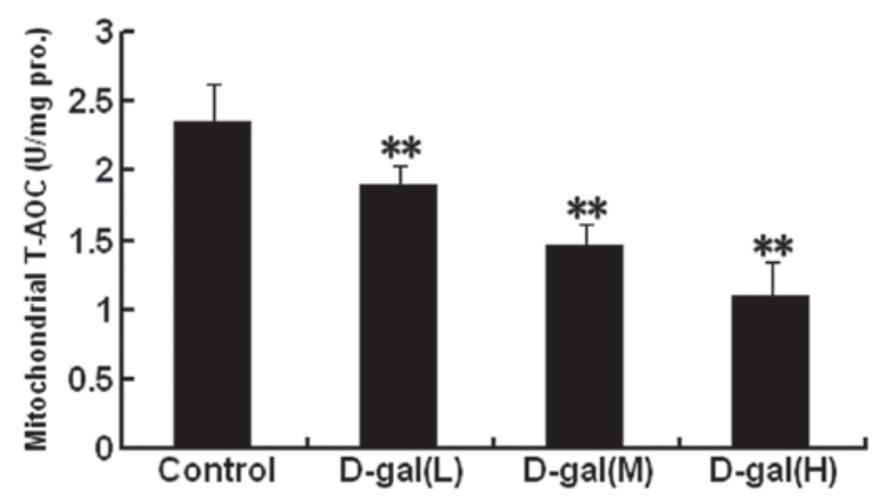

Figure 4. Levels of mitochondrial T-AOC in the auditory cortex of rats in the different groups. The mitochondrial T-AOC levels were significantly decreased in the D-gal-treated groups as compared with those in the control group. Data are expressed as the mean \pm standard deviation of six rats per group. ${ }^{* *} \mathrm{P}<0.01$ versus the control group. $\mathrm{D}$-gal (L), low-dose $\mathrm{D}$-gal group; D-gal (M), medium-dose D-gal group; D-gal (H), high-dose D-gal group; T-AOC, total antioxidant capability, D-gal, D-galactose.

\section{Discussion}

Oxidative damage to mtDNA has a strong association with the molecular process of aging (22). To the best of our knowledge, this study showed for the first time that levels of NOX2 and $8-\mathrm{OHdG}$, a biomarker of DNA oxidative damage, were significantly increased in the auditory cortex of the CAS in rats with D-gal-induced aging. The expression of $8-\mathrm{OHdG}$ was observed predominantly in the cytoplasm of cells, suggesting that D-gal induced mtDNA oxidative damage in the auditory cortex. These findings indicate that D-gal may induce mtDNA oxidative damage in the CAS in part through the NOX2 pathway, and NOX2-associated ROS generation may play an essential role in the aging process of the CAS. NOXs function to produce ROS, as opposed to mitochondria, which generate ROS as a byproduct of their metabolism (23). Previous

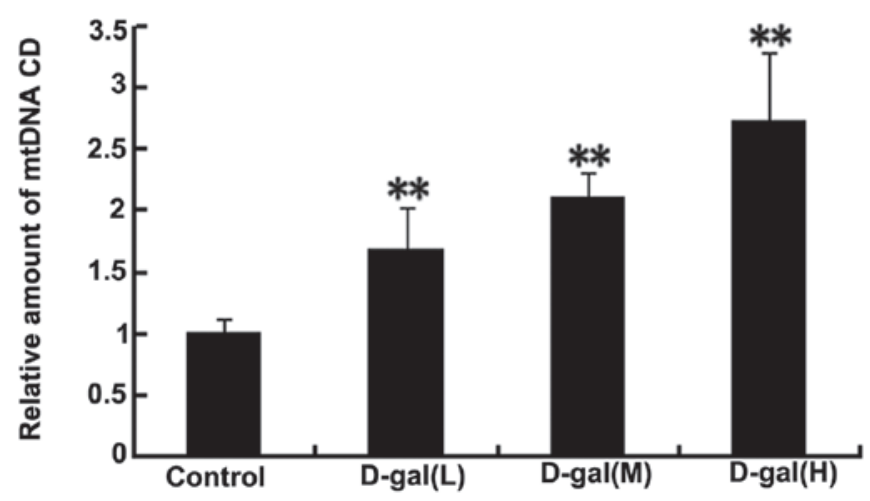

Figure 5. Quantitative analysis of the accumulation of the mtDNA CD in the auditory cortex of rats in the different groups. The levels of the mtDNA CD were significantly increased in the D-gal-treated groups as compared with those in the control group. Data are expressed as the mean \pm standard deviation of six rats per group. ${ }^{* *} \mathrm{P}<0.01$ versus the control group. mtDNA, mitochondrial DNA; CD, common deletion; D-gal (L), low-dose D-gal group; D-gal (M), medium-dose D-gal group; D-gal (H), high-dose D-gal group; D-gal, D-galactose.

studies have demonstrated that NOX3 represents the primary source of ROS generation in the inner ear, which contributes to cisplatin-induced ROS generation in the PAS $(13,24,25)$. It has additionally been shown that NOX3-associated ROS generation may function in the degeneration in the PAS (5), and NOX2-dependent oxidative stress may contribute to the mtDNA CD and mitochondrial ultrastructural damage in the hippocampus (26) of rats with D-gal-induced aging. Therefore, NOX2-associated ROS generation may be an important source of ROS in the aging process of the CAS.

Mitochondria are another important source of ROS in cells (16). UCP2, which is located in the mitochondrial inner membrane, can act to slightly lower the proton-motive force across the membrane through a mild uncoupling and, in this way, attenuate mitochondrial ROS generation (17). In a 
previous study, it was demonstrated that UCP2 mRNA expression was upregulated in the inner ear ganglia cells of the mouse following aminoglycoside intoxication, and these responses were blocked by a co-administration with antioxidant (27). Previous studies have also reported that UCP 2 mRNA expression in the vestibular ganglion of the inner ear was significantly upregulated following a labyrinthectomy (28), and UCP2 mRNA and protein expression was significantly increased in the inner ear of D-gal- and/or high-fat diet-treated rats (5). These previous studies have indicated that UCP2 may indirectly reflect the ROS levels of cells and play a protective role against oxidative damage in the inner ear. In the present study, UCP2 was found to be overexpressed in the auditory cortex of rats with D-gal-induced aging, which may also suggest that increased UCP2 levels reflect increases in ROS generation and that UCP2 plays protective roles in the aging process of the $\mathrm{CAS}$ in rats with D-gal-induced aging.

mtDNA is highly susceptible to ROS-induced damage due to its close proximity to the sites of ROS generation and lack of protective histones (29). Increases in ROS generation together with decreases in mitochondrial T-AOCs may induce mtDNA oxidative damage. The most common type of mtDNA damage associated with aging is the mtDNA 4,977-bp deletion (also known as the $\mathrm{CD}$ ) in humans and the corresponding mtDNA 4,834-bp deletion in rats. Therefore, the mtDNA CD has been widely used as a biomarker for aging (21,30-32). An association between elevated mtDNA CD levels and ARHL has been observed in a number of studies (32-34). Previous studies have demonstrated that the frequency of the mtDNA CD was increased in the PAS $(5,6,8,10,11)$ and CAS $(4,7,9)$ of D-gal-induced aging rats. The present study also found that the mtDNA CD levels were increased in the auditory cortex of D-gal-treated rats, and these increased mtDNA CD levels correlated with mtDNA oxidative damage. Therefore, these findings suggest that mtDNA damage in the auditory cortex of rats with D-gal-induced aging may be caused by NOX2- and mitochondria-associated ROS generation.

In conclusion, the present findings indicate that both NOXand mitochondria-associated ROS generation may contribute to mtDNA oxidative damage in the auditory cortex of the CAS of rats with D-gal-induced aging. NOX2 and UCP2 may therefore be useful therapeutic targets to prevent or slow the development of ARHL.

\section{Acknowledgements}

This study was supported by grants from the Shenzhen Nanshan Science and Technology Development Foundation (no. 2012014) and the National Natural Science Foundation of China (no. 61302037).

\section{References}

1. Frisina RD and Walton JP: Age-related structural and functional changes in the cochlear nucleus. Hear Res 216-217: 216-223, 2006.

2. Howarth A and Shone GR: Ageing and the auditory system. Postgrad Med J 82: 166-171, 2006.

3. Wu L, Sun Y, Hu YJ, et al: Increased p66Shc in the inner ear of D-galactose-induced aging mice with accumulation of mitochondrial DNA 3873-bp deletion: p66Shc and mtDNA damage in the inner ear during aging. PLoS One 7: e50483, 2012.
4. Zhong Y, Hu Y, Peng W, et al: Age-related decline of the cytochrome $c$ oxidase subunit expression in the auditory cortex of the mimetic aging rat model associated with the common deletion. Hear Res 294: 40-48, 2012.

5. Du Z, Yang Y, Hu Y, et al: A long-term high-fat diet increases oxidative stress, mitochondrial damage and apoptosis in the inner ear of D-galactose-induced aging rats. Hear Res 287: 15-24, 2012.

6. Zhong Y, Hu YJ, Chen B, et al: Mitochondrial transcription factor A overexpression and base excision repair deficiency in the inner ear of rats with D-galactose-induced aging. FEBS J 278: 2500-2510, 2011.

7. Chen B, Zhong Y, Peng W, et al: Increased mitochondrial DNA damage and decreased base excision repair in the auditory cortex of D-galactose-induced aging rats. Mol Biol Rep 38: 3635-3642, 2011.

8. Zhong Y, Hu YJ, Yang Y, et al: Contribution of common deletion to total deletion burden in mitochondrial DNA from inner ear of d-galactose-induced aging rats. Mutat Res 712: 11-19, 2011.

9. Chen B, Zhong Y, Peng W, Sun Y and Kong WJ: Age-related changes in the central auditory system: comparison of D-galactose-induced aging rats and naturally aging rats. Brain Res 1344: 43-53, 2010.

10. Kong WJ, Wang Y, Wang Q, Hu YJ, Han YC and Liu J: The relation between D-galactose injection and mitochondrial DNA 4834 bp deletion mutation. Exp Gerontol 41: 628-634, 2006.

11. Kong WJ, Hu YJ, Wang Q, et al: The effect of the mtDNA4834 deletion on hearing. Biochem Biophys Res Commun 344: 425-430, 2006.

12. Bedard K and Krause KH: The NOX family of ROS-generating NADPH oxidases: physiology and pathophysiology. Physiol Rev 87: 245-313, 2007.

13. Bánfi B, Malgrange B, Knisz J, Steger K, Dubois-Dauphin M and Krause KH: NOX3, a superoxide-generating NADPH oxidase of the inner ear. J Biol Chem 279: 46065-46072, 2004.

14. Quinn MT, Ammons MC and Deleo FR: The expanding role of NADPH oxidases in health and disease: no longer just agents of death and destruction. Clin Sci (Lond) 111: 1-20, 2006.

15. Serrano F, Kolluri NS, Wientjes FB, Card JP and Klann E: NADPH oxidase immunoreactivity in the mouse brain. Brain Res 988: 193-198, 2003.

16. Raha $\mathrm{S}$ and Robinson BH: Mitochondria, oxygen free radicals, disease and ageing. Trends Biochem Sci 25: 502-508, 2000.

17. Brand MD and Esteves TC: Physiological functions of the mitochondrial uncoupling proteins UCP2 and UCP3. Cell Metab 2: 85-93, 2005.

18. Kujoth GC, Hiona A, Pugh TD, et al: Mitochondrial DNA mutations, oxidative stress, and apoptosis in mammalian aging. Science 309: 481-484, 2005.

19. Ma Y, Mehta SL, Lu B and Li PA: Deficiency in the inner mitochondrial membrane peptidase 2-like (Immp21) gene increases ischemic brain damage and impairs mitochondrial function. Neurobiol Dis 44: 270-276, 2011.

20. Livak KJ and Schmittgen TD: Analysis of relative gene expression data using real-time quantitative PCR and the 2(-Delta Delta C(T)) Method. Methods 25: 402-408, 2001.

21. Nicklas JA, Brooks EM, Hunter TC, Single R and Branda RF: Development of a quantitative PCR (TaqMan) assay for relative mitochondrial DNA copy number and the common mitochondrial DNA deletion in the rat. Environ Mol Mutagen 44: 313-320, 2004.

22. Wang $\mathrm{CH}$, Wu SB, Wu YT and Wei YH: Oxidative stress response elicited by mitochondrial dysfunction: implication in the pathophysiology of aging. Exp Biol Med (Maywood) 238: 450-460, 2013

23. Krause KH: Aging: a revisited theory based on free radicals generated by NOX family NADPH oxidases. Exp Gerontol 42: 256-262, 2007.

24. Mukherjea D, Jajoo S, Kaur T, Sheehan KE, Ramkumar V and Rybak LP: Transtympanic administration of short interfering (si)RNA for the NOX3 isoform of NADPH oxidase protects against cisplatin-induced hearing loss in the rat. Antioxid Redox Signal 13: 589-598, 2010.

25. Mukherjea D, Jajoo S, Sheehan K, et al: NOX3 NADPH oxidase couples transient receptor potential vanilloid 1 to signal transducer and activator of transcription 1-mediated inflammation and hearing loss. Antioxid Redox Signal 14: 999-1010, 2011.

26. Du Z, Hu Y, Yang Y, et al: NADPH oxidase-dependent oxidative stress and mitochondrial damage in hippocampus of D-galactose-induced aging rats. J Huazhong Univ Sci Technolog Med Sci 32: 466-472, 2012. 
27. Kitahara T, Li-Korotky HS and Balaban CD: Regulation of mitochondrial uncoupling proteins in mouse inner ear ganglion cells in response to systemic kanamycin challenge. Neuroscience 135 : 639-653, 2005

28. Kitahara T, Horii A, Kizawa K, Maekawa C and Kubo T: Changes in mitochondrial uncoupling protein expression in the rat vestibular nerve after labyrinthectomy. Neurosci Res 59: 237-242, 2007.

29. Druzhyna NM, Wilson GL and LeDoux SP: Mitochondrial DNA repair in aging and disease. Mech Ageing Dev 129: 383-390, 2008

30. Meissner C, Bruse P, Mohamed SA, et al: The 4977 bp deletion of mitochondrial DNA in human skeletal muscle, heart and different areas of the brain: a useful biomarker or more? Exp Gerontol 43: 645-652, 2008.
31. Yowe DL and Ames BN: Quantitation of age-related mitochondrial DNA deletions in rat tissues shows that their pattern of accumulation differs from that of humans. Gene 209: 23-30, 1998.

32. Markaryan A, Nelson EG and Hinojosa R: Quantification of the mitochondrial DNA common deletion in presbycusis. Laryngoscope 119: 1184-1189, 2009.

33. Bai U, Seidman MD, Hinojosa R and Quirk WS: Mitochondrial DNA deletions associated with aging and possibly presbycusis: a human archival temporal bone study. Am J Otol 18: 449-453, 1997.

34. Ueda N, Oshima T, Ikeda K, Abe K, Aoki M and Takasaka T: Mitochondrial DNA deletion is a predisposing cause for sensorineural hearing loss. Laryngoscope 108: 580-584, 1998. 